\title{
Erratum: Integrability and duality in spin chains [Phys. Rev. B 99, 075111 (2019)]
}

Eyzo Stouten, Pieter W. Claeys, Jean-Sébastien Caux, and Vladimir Gritsev

(C) (Received 18 March 2019; published 12 April 2019)

DOI: 10.1103/PhysRevB.99.169902

It was brought to our attention that another general solution to the Yang-Baxter equation was proposed by Martins [1] in the context of vertex models.

[1] M. J. Martins, The symmetric six-vertex model and the Segre cubic threefold, J. Phys. A: Math. Theor. 48, 334002 (2015). 